\title{
A FUNCTIONAL-ANALYTIC METHOD FOR THE STUDY OF DIFFERENCE EQUATIONS
}

\author{
EUGENIA N. PETROPOULOU AND PANAYIOTIS D. SIAFARIKAS
}

Received 29 October 2003 and in revised form 10 February 2004

We will give the generalization of a recently developed functional-analytic method for studying linear and nonlinear, ordinary and partial, difference equations in the $\ell_{p}^{1}$ and $\ell_{p}^{2}$ spaces, $p \in \mathbb{N}, p \geq 1$. The method will be illustrated by use of two examples concerning a nonlinear ordinary difference equation known as the Putnam equation, and a linear partial difference equation of three variables describing the discrete Newton law of cooling in three dimensions.

\section{Introduction}

The aim of this paper is to present the generalization of a functional-analytic method, which was recently developed for the study of linear and nonlinear difference equations of one, two, three, and four variables in the Hilbert space

$$
\ell_{p}^{2}=\left\{f\left(i_{1}, \ldots, i_{p}\right): \mathbb{N}^{p} \longrightarrow \mathbb{C}: \sum_{i_{1}=1}^{\infty} \cdots \sum_{i_{p}=1}^{\infty}\left|f\left(i_{1}, \ldots, i_{p}\right)\right|^{2}<+\infty\right\}
$$

and the Banach space

$$
\ell_{p}^{1}=\left\{f\left(i_{1}, \ldots, i_{p}\right): \mathbb{N} \longrightarrow \mathbb{C}: \sum_{i_{1}=1}^{\infty} \cdots \sum_{i_{p}=1}^{\infty}\left|f\left(i_{1}, \ldots, i_{p}\right)\right|<+\infty\right\}
$$

where $\mathbb{N} p=\underbrace{\mathbb{N} \times \cdots \times \mathbb{N}}_{p \text {-times }}$ and $p=1,2,3,4$.

More precisely, this method was introduced for the first time by Ifantis in [5] for the study of linear and nonlinear ordinary difference equations. Later, this method was extended by the authors in $[7,9,10]$ in order to study a class of nonlinear ordinary difference equations more general than the one studied in [5]. For the study of linear and 
nonlinear partial difference equations of two variables, we developed a similar functionalanalytic method in $[11,12]$, which was extended in [8] in order to study partial difference equations of three and four variables.

The aim of this paper is to present the generalization of this functional-analytic method for the study of linear and nonlinear partial difference equations of $p$ variables in the Hilbert space $\ell_{p}^{2}$, defined by (1.1), and the Banach space $\ell_{p}^{1}$, defined by (1.2), respectively, with $p \in \mathbb{N}, p \geq 1$. The motivation for seeking solutions of partial difference equations in the spaces $\ell_{p}^{2}$ and $\ell_{p}^{1}$ arises from various problems of mathematics, physics, and biology, such as probability problems, problems concerning integral equations, generating analytic functions, Laurent or $z$-transforms, numerical schemes, boundary value problems of partial differential equations, problems of quantum mechanics, and problems of population dynamics and epidemiology (for more details, see [11] and the references therein). Also, by assuring the existence of a solution of a difference equation in the space $\ell_{p}^{2}$ or $\ell_{p}^{1}$, we obtain information regarding the asymptotic behavior of the unknown sequence for initial conditions which are in general complex numbers.

We would like, at this point, to give an outline of the functional-analytic method that we will present in details in Section 2. (For a sketch of the main ideas used in the proofs of our main results, see the beginning of Section 3.) By use of this method, the linear or nonlinear difference equation under consideration is transformed equivalently into a linear or nonlinear operator equation defined in an abstract Hilbert space $H$ or Banach space $H_{1}$, respectively. In this way, we can use various results (e.g., fixed point theorems) from the wealth of operator theory, in order to assure the existence of a unique solution of the operator equation in $H$ or $H_{1}$. In the case of linear equations, we use the following classical result of operator theory [4, pages 70-71].

Theorem 1.1. Let $T$ be a linear, bounded operator of the Hilbert space $H$ with $\|T\|<1$. Then the inverse of $I-T$ exists on $H$ and is uniquely determined and bounded by $\|(I-$ $T)^{-1} \| \leq 1 /(1-\|T\|)$.

In the case of nonlinear equations, we use the following fixed point theorem of Earle and Hamilton [3].

Theorem 1.2. Let $X$ be a bounded, connected, and open subset of a Banach space B. Further, let $g: X \rightarrow g(X)$ be holomorphic, that is, its Fréchet derivative exists and $g(X)$ lies strictly inside $X$. Then $g$ has a unique fixed point in $X$. (By saying that a subset $X^{\prime}$ of $X$ lies strictly inside $X$, we mean that there exists $\epsilon>0$ such that $\left\|x^{\prime}-y\right\|>\epsilon$ for all $x^{\prime} \in X^{\prime}$ and $y \in$ $B-X$.)

For both linear and nonlinear difference equations, we obtain, by use of our method, a bound of the solution of the difference equation under consideration. Moreover, in the case of nonlinear difference equations, we use a constructive technique, which allows us to obtain a region, depending on the initial conditions and the parameters of the equations, where the solution of the difference equation under consideration holds.

We illustrate our method in Section 3 by applying it to two difference equations which arise from a mathematical problem (the Putnam equation) and a physical problem concerning the discrete Newton law of cooling in three dimensions. 


\section{The functional-analytic method}

We denote by $H$ an abstract separable Hilbert space with orthonormal base $\left\{e_{i_{1}, \ldots, i_{p}}\right\}$, $i_{1}, \ldots, i_{p}=1,2, \ldots$, and elements $u \in H$ which have the form

$$
u=\sum_{i_{1}=1}^{\infty} \cdots \sum_{i_{p}=1}^{\infty}\left(u, e_{i_{1}, \ldots, i_{p}}\right) e_{i_{1}, \ldots, i_{p}}
$$

with norm $\|u\|^{2}=\sum_{i_{1}=1}^{\infty} \cdots \sum_{i_{p}=1}^{\infty}\left|\left(u, e_{i_{1}, \ldots, i_{p}}\right)\right|^{2}$. Also, by $H_{1}$ we mean the Banach space consisting of those elements $u \in H$ which satisfy the condition

$$
\sum_{i_{1}=1}^{\infty} \cdots \sum_{i_{p}=1}^{\infty}\left|\left(u, e_{i_{1}, \ldots, i_{p}}\right)\right|<+\infty .
$$

The norm in $H_{1}$ is denoted by $\|u\|_{1}=\sum_{i_{1}=1}^{\infty} \cdots \sum_{i_{p}=1}^{\infty}\left|\left(u, e_{i_{1}, \ldots, i_{p}}\right)\right|$. By $u\left(i_{1}, \ldots, i_{p}\right)$ we mean an element of $l_{p}^{2}$ or $l_{p}^{1}$, and by $u=\sum_{i_{1}=1}^{\infty} \cdots \sum_{i_{p}=1}^{\infty}\left(u, e_{i_{1}, \ldots, i_{p}}\right) e_{i_{1}, \ldots, i_{p}}$ we mean that element of $H$ or $H_{1}$ generated by $u\left(i_{1}, \ldots, i_{p}\right)$.

Finally, we define in $H$ the shift operators $V_{j}, j=1, \ldots, p$, as follows:

$$
V_{j} e_{i_{1}, \ldots, i_{j}, \ldots, i_{p}}=e_{i_{1}, \ldots, i_{j}+1, \ldots, i_{p}} .
$$

It can be easily seen that their adjoint operators are

$$
V_{j}^{*} e_{i_{1}, \ldots, i_{j}, \ldots, i_{p}}=e_{i_{1}, \ldots, i_{j}-1, \ldots, i_{p}}, \quad i_{j}=2,3, \ldots, \quad V_{j}^{*} e_{i_{1}, \ldots, 1, \ldots, i_{p}}=0,
$$

and that

$$
\left\|V_{j}^{*}\right\|=\left\|V_{j}\right\|=\left\|V_{j}^{*}\right\|_{1}=\left\|V_{j}\right\|_{1}=1, \quad j=1, \ldots, p .
$$

The following proposition is of fundamental importance in our approach.

Proposition 2.1. The function

$$
\phi: H \longrightarrow l_{p}^{2}, \quad \phi(u)=\left(u, e_{i_{1}, \ldots, i_{p}}\right)=u\left(i_{1}, \ldots, i_{p}\right),
$$

is an isomorphism from $\mathrm{H}$ onto $l_{p}^{2}$.

Proof. We begin by showing that the mapping defined by (2.6) is well defined. Indeed, since $u \in H$, we have

$$
\begin{aligned}
\left\|u\left(i_{1}, \ldots, i_{p}\right)\right\|_{i_{p}^{2}}^{2} & =\sum_{i_{1}=1}^{\infty} \cdots \sum_{i_{p}=1}^{\infty}\left|u\left(i_{1}, \ldots, i_{p}\right)\right|^{2} \\
& =\sum_{i_{1}=1}^{\infty} \cdots \sum_{i_{p}=1}^{\infty}\left|\left(u, e_{i_{1}, \ldots, i_{p}}\right)\right|^{2} \\
& =\|u\|^{2}<+\infty .
\end{aligned}
$$


By use of the properties of an inner product, it is obvious that $\phi$ is linear. Also, $\phi$ is a one-to-one mapping onto $l_{p}^{2}$. Indeed, if $u \in H, v \in H$ with $\phi(u)=\phi(v)$, then

$$
\left(u-v, e_{i_{1}, \ldots, i_{p}}\right)=0 \Longleftrightarrow u=v,
$$

because $e_{i_{1}, \ldots, i_{p}}$ is an orthonormal base of $H$.

Furthermore, if $\alpha\left(i_{1}, \ldots, i_{p}\right) \in l_{p}^{2}$, then there exists $u \in H$ such that $\phi(u)=\alpha\left(i_{1}, \ldots, i_{p}\right)$. This $u$ is given by

$$
u=\sum_{i_{1}=1}^{\infty} \cdots \sum_{i_{p}=1}^{\infty} \alpha\left(i_{1}, \ldots, i_{p}\right) e_{i_{1}, \ldots, i_{p}}
$$

and it belongs to $H$ since

$$
\|u\|^{2}=\sum_{i_{1}=1}^{\infty} \cdots \sum_{i_{p}=1}^{\infty}\left|\alpha\left(i_{1}, \ldots, i_{p}\right)\right|^{2}=\left\|\alpha\left(i_{1}, \ldots, i_{p}\right)\right\|_{l_{p}^{2}}^{2}<+\infty .
$$

Finally, the mapping $\phi$ preserves the norm since

$$
\|\phi(u)\|^{2}=\sum_{i_{1}=1}^{\infty} \cdots \sum_{i_{p}=1}^{\infty}\left|u\left(i_{1}, \ldots, i_{p}\right)\right|^{2}=\sum_{i_{1}=1}^{\infty} \cdots \sum_{i_{p}=1}^{\infty}\left|\left(u, e_{i_{1}, \ldots, i_{p}}\right)\right|^{2}=\|u\|^{2} .
$$

Thus, the mapping $\phi$ defined by (2.6) is an isomorphism from $H$ onto $l_{p}^{2}$.

In a similar way, the following proposition can also be proved.

Proposition 2.2. The function

$$
\phi: H \longrightarrow l_{p}^{1}, \quad \phi(u)=\left(u, e_{i_{1}, \ldots, i_{p}}\right)=u\left(i_{1}, \ldots, i_{p}\right),
$$

is an isomorphism from $H$ onto $l_{p}^{1}$.

We call the element $u$, defined by (2.6) or (2.12), the abstract form of $u\left(i_{1}, \ldots, i_{p}\right)$ in $H$ or $H_{1}$, respectively. In general, if $G$ is a mapping in $l_{p}^{2}\left(l_{p}^{1}\right)$ and $N$ is a mapping in $H\left(H_{1}\right)$, we call $N(u)$ the abstract form of $G\left(u\left(i_{1}, \ldots, i_{p}\right)\right)$ if

$$
G\left(u\left(i_{1}, \ldots, i_{p}\right)\right)=\left(N(u), e_{i_{1}, \ldots, i_{p}}\right) .
$$

\section{Illustrative examples}

In this section, we will illustrate our method using two characteristic examples of difference equations arising in a problem of mathematics and a problem of physics. More precisely, we will establish conditions so that the difference equations under consideration have a unique bounded solution in $l_{p}^{1}$ or $l_{p}^{2}$. Such kind of solutions is extremely useful not only from a mathematical point of view, but also from an applied point of view (see Remarks 3.2 and 3.4). 
We would like now to give the main ideas used in the proofs of our results. First, using (2.6) or (2.12), we transform the linear or nonlinear difference equation under consideration into an equivalent linear or nonlinear operator equation in an abstract separable Hilbert $H$ or Banach $H_{1}$ space. Then, after some manipulations, we bring the linear operator equation into the form

$$
(I-T) u=f
$$

where $u \in H$ is the unknown variable, $f$ a known element of $H$, and $T: H \rightarrow H$ a known linear operator. At this point, we impose conditions so that $\|T\|<1$, in order to apply Theorem 1.1 to the preceding operator equation and obtain information for the initial linear difference equation under consideration.

In the case of nonlinear equations, we do some manipulation in order to write the operator equation in the form

$$
u=g(u)
$$

where $u \in H$ is the unknown variable and $g: X \subset H_{1} \rightarrow g(X)$ a known nonlinear mapping. Usually, $g(u)$ has the form

$$
g(u)=h+\phi(u)
$$

where $h$ is a known element of $H_{1}$ depending on the initial conditions and the nonhomogeneous term (if any) of the initial nonlinear difference equation, and $\phi: H_{1} \rightarrow H_{1}$ is a known nonlinear mapping. At this point, we impose conditions on $\|h\|_{1}$ in order to apply the fixed point Theorem 1.2 to equation $u=g(u)$ and obtain information for the initial nonlinear difference equation under consideration.

3.1. The Putnam equation. Consider the nonlinear, homogeneous, ordinary difference equation

$$
\begin{aligned}
f(i+3)+f(i+2)= & f(i+4) f(i+3) f(i+2)+f(i+4) f(i+1) \\
& +f(i+4) f(i)-f(i+1) f(i), \quad i=1,2, \ldots
\end{aligned}
$$

Equation (3.4) appeared in a problem given in the 25th William Lowell Putnam Mathematical Competition, held on December 5, 1964 (see [1]). This problem is as follows [1]:

"Let $p_{n}, n=1,2, \ldots$, be a bounded sequence of integers, which satisfies the recursion

$$
p_{n}=\frac{p_{n-1}+p_{n-2}+p_{n-3} p_{n-4}}{p_{n-1} p_{n-2}+p_{n-3}+p_{n-4}}
$$

Show that the sequence eventually becomes periodic."

As mentioned in [1], the solution of this problem is independent of the recurrence relation that the sequence $p_{n}$ satisfies, as long as $p_{n}$ is bounded. In the years that passed, it turned out that (3.5) is quite attractive from a mathematical point of view. In this paper, we will prove the following result. 
Result 3.1. The Putnam equation (3.4) has a unique bounded solution in $\ell_{1}^{1}+\{1\}$ if

$$
|f(1)-1|+|f(2)-1|+|f(3)-1|+|f(4)-1|<0.120227
$$

which satisfies

$$
|f(i)|<1.236068
$$

where the initial conditions $f(1), f(2), f(3)$, and $f(4)$ are in general complex numbers.

Remark 3.2. (a) It is obvious from the preceding result that the solution of the Putnam equation (3.4) tends to 1 if (3.6) holds. Thus, 1 is a locally asymptotically stable equilibrium point of (3.4) if (3.6) holds.

(b) In [6], it was proved, among other things, that the equilibrium point 1 of (3.4) is globally asymptotically stable for positive initial conditions.

Proof of Result 3.1. Equation (3.4) is a nonlinear ordinary difference equation, that is, a difference equation of $p=1$ variable. As a consequence, we will work in the Banach space $\ell_{1}^{1}$ and the isomorphic abstract Banach space $H_{1}$ with orthonormal base $\left\{e_{i}\right\}, i=1,2, \ldots$ (For reasons of simplicity, we will use the symbol $i$ instead of the symbol $i_{1}$.)

First of all, we mention that $\varrho=1$ is an equilibrium point of (3.4) and we set $f(i)=$ $u(i)+\varrho$. Then (3.4) becomes

$$
\begin{aligned}
\left(\varrho^{2}+2 \varrho\right) & u(i+4)+\left(\varrho^{2}-1\right) u(i+3)+\left(\varrho^{2}-1\right) u(i+2) \\
= & -u(i+4) u(i+1)-u(i+4) u(i+3) u(i+2)-u(i+4) u(i) \\
& +u(i+1) u(i)-\varrho u(i+4) u(i+3)-\varrho u(i+4) u(i+2)-\varrho u(i+3) u(i+2) .
\end{aligned}
$$

Using (2.12), we find the abstract forms of all the terms involved in (3.8). More precisely, we have

$$
\begin{gathered}
u(i+k)=\left(u, e_{i+k}\right)=\left(u, V_{1}^{k} e_{i}\right)=\left(\left(V_{1}^{*}\right)^{k} u, e_{i}\right), \quad k=2,3,4, \\
u(i+m) u(i+n)=\left(u, e_{i+m}\right)\left(u, e_{i+n}\right) e_{i}=N_{m n}(u), \quad m, n=0,1,2,3,4, \\
u(i+4) u(i+3) u(i+2)=\left(u, e_{i+4}\right)\left(u, e_{i+3}\right)\left(u, e_{i+2}\right) e_{i}=N_{2}(u) .
\end{gathered}
$$

Moreover, we can prove that the nonlinear operators $N_{m n}(u), N_{2}(u)$ are Frechét-differentiable in $H_{1}$. Thus, the abstract form of (3.8) in $H_{1}$ is

$$
\begin{aligned}
& \left(\varrho^{2}+2 \varrho\right)\left(V_{1}^{*}\right)^{4} u+\left(\varrho^{2}-1\right)\left(V_{1}^{*}\right)^{3} u+\left(\varrho^{2}-1\right)\left(V_{1}^{*}\right)^{2} u \\
& \quad=-N_{41}(u)-N_{2}(u)-N_{40}(u)+N_{10}(u)-\varrho N_{43}(u)-\varrho N_{42}(u)-\varrho N_{32}(u) \Longrightarrow\left(V_{1}^{*}\right)^{4} u \\
& \quad=-\frac{1}{3} N_{41}(u)-\frac{1}{3} N_{2}(u)-\frac{1}{3} N_{40}(u)+\frac{1}{3} N_{10}(u)-\frac{1}{3} N_{43}(u)-\frac{1}{3} N_{42}(u)-\frac{1}{3} N_{32}(u)
\end{aligned}
$$


or, due to the fact that $V^{*} e_{1}=0$,

$$
\begin{aligned}
u= & g(u) \\
= & u(1) e_{1}+u(2) e_{2}+u(3) e_{3}+u(4) e_{4} \\
& -\frac{1}{3} V^{4}\left[N_{41}(u)+N_{2}(u)+N_{40}(u)-N_{10}(u)+N_{43}(u)+N_{42}(u)+N_{32}(u)\right] .
\end{aligned}
$$

From the preceding equation we obtain, taking the norm of both parts in $H_{1}$,

$$
\begin{aligned}
\|u\|_{1}= & \|g(u)\|_{1} \\
\leq & |u(1)|+|u(2)|+|u(3)|+|u(4)| \\
& +\frac{1}{3}\left[\left\|N_{41}(u)\right\|_{1}+\left\|N_{2}(u)\right\|_{1}+\left\|N_{40}(u)\right\|_{1}+\left\|N_{10}(u)\right\|_{1}\right. \\
& \left.\quad+\left\|N_{43}(u)\right\|_{1}+\left\|N_{42}(u)\right\|_{1}+\left\|N_{32}(u)\right\|_{1}\right] \Longrightarrow\|u\|_{1} \\
\leq & |u(1)|+|u(2)|+|u(3)|+|u(4)|+\frac{1}{3}\left(\|u\|_{1}^{3}+6\|u\|_{1}^{2}\right) .
\end{aligned}
$$

Let $\|u\|_{1} \leq R, R$ sufficiently large but finite. Then, from (3.12), we have

$$
\|u\|_{1} \leq|u(1)|+|u(2)|+|u(3)|+|u(4)|+\frac{1}{3} R^{3}+2 R^{2} .
$$

Let $P(R)=R-2 R^{2}-(1 / 3) R^{3}$. This function has a maximum at $R_{0}=\sqrt{5}-2 \cong 0.236068$, which is $P_{0} \cong 0.120227$. Thus, for $R=R_{0}$, we find that if

$$
|u(1)|+|u(2)|+|u(3)|+|u(4)| \leq P_{0}-\epsilon, \quad \epsilon>0,
$$

then

$$
\|g(u)\|_{1} \leq R_{0}-\epsilon<R_{0}
$$

for $\|u\|_{1}<R_{0}$. This means that for

$$
|u(1)|+|u(2)|+|u(3)|+|u(4)|<P_{0}
$$

$g$ is a holomorphic mapping from $X=B\left(0, R_{0}\right)=\left\{u \in H_{1}:\|u\|_{1}<R_{0}\right\}$ strictly inside $X=$ $B\left(0, R_{0}\right)$. Indeed, it is obvious that $g(X) \subseteq X$. Moreover, $g(X)$ lies strictly inside $X$, since if $w \in H_{1}-X \Rightarrow\|w\|_{1} \geq R_{0}$ and $w^{\prime} \in g(X)$, that is, there exists an $f \in X \Rightarrow\|f\|_{1}<R_{0}$ such that $g(f)=w^{\prime}$, then we find easily that $\left\|w-w^{\prime}\right\| \geq \epsilon>\epsilon / 2=\epsilon_{1}$. As a consequence, the fixed point theorem of Earle and Hamilton can be applied to (3.11). Thus, for

$$
|u(1)|+|u(2)|+|u(3)|+|u(4)|<P_{0}
$$




\section{Functional-analytic method for difference equations}

(3.11) has a unique solution in $H_{1}$ bounded by $R_{0}$. Equivalently, this means that if (3.17) holds, then the difference equation (3.8) has a unique solution in $\ell_{1}^{1}$ bounded by $R_{0}$. As a consequence, if (3.6) holds, (3.4) has a unique solution in $\ell_{1}^{1}+\{1\}$ bounded by $1+R_{0}$.

\subsection{A linear difference equation of three variables describing the discrete Newton law} of cooling. Consider the linear, homogeneous, partial difference equation

$$
\begin{aligned}
& u(i, j, n+1)+[4 r(i, j, n)-1] u(i, j, n)-r(i, j, n) u(i-1, j, n) \\
& -r(i, j, n) u(i+1, j, n)-r(i, j, n) u(i, j-1, n)-r(i, j, n) u(i, j+1, n)=0,
\end{aligned}
$$

where $i, j, n=1,2, \ldots$, and $r(i, j, n)$ is a known sequence. Equation (3.18) describes the discrete Newton law of cooling in three dimensions. More precisely, the physical problem that (3.18) describes is the following.

Consider the distribution of heat through a "very long" (so long that it can be labelled by the set of integers) nonuniform thin plate. Let $u(i, j, n)$ be the temperature of the plate at the position $(i, j)$ and time $n$. At time $n$, if the temperature $u(i-1, j, n)$ is higher than $u(i, j, n)$, heat will flow from the point $(i-1, j)$ to $(i, j)$ at a rate $r(i, j, n)$. Similarly, heat will flow from the point $(i+1, j)$ to $(i, j)$ at the same rate, $r(i, j, n)$. Thus, the total effect will be

$$
\begin{aligned}
u(i, j, n+1)-u(i, j, n)= & r(i, j, n)[u(i-1, j, n)-2 u(i, j, n)+u(i+1, j, n)] \\
& +r(i, j, n)[u(i, j-1, n)-2 u(i, j, n)+u(i, j+1, n)],
\end{aligned}
$$

which is essentially (3.18). For (3.18), bounded and/or positive solutions of (3.18) are of interest (see [2]). In this paper, we will prove the following result.

Result 3.3. (a) Let

$$
\begin{gathered}
\sup _{i, j, n}\left|\frac{1}{4 r(i, j, n)-1}\right|<+\infty, \\
\sup _{i, j, n}\left|\frac{1}{4 r(i, j, n)-1}\right|\left[1+4 \sup _{i, j, n}|r(i, j, n)|\right]<1 .
\end{gathered}
$$

Then the unique solution of (3.18) in $\ell_{3}^{2}$ is the zero solution.

(b) Let

$$
\sup _{i, j, n}|4 r(i, j, n)-1|+4 \sup _{i, j, n}|r(i, j, n)|<1 .
$$


Then (3.18) has a unique bounded solution in $\ell_{3}^{2}$, which satisfies

$$
|u(i, j, n)| \leq \frac{\|u(i, j, 1)\|_{\ell_{\mathbb{N}^{2}}^{2}}}{1-\sup _{i, j, n}|4 r(i, j, n)-1|-4 \sup _{i, j, n}|r(i, j, n)|},
$$

provided that the initial conditions $u(i, j, 1)$ (which are in general complex) belong to $\ell_{2}^{2}$.

Proof of Result 3.3. Equation (3.18) is a linear partial difference equation of $p=3$ variables. As a consequence, we will work in the Hilbert space $\ell_{3}^{2}$ and the isomorphic abstract Hilbert space $H$ with orthonormal base $\left\{e_{i, j, n}\right\}, i, j, n=1,2, \ldots$ (For reasons of simplicity, we will use the symbols $i, j$, and $n$ instead of the symbols $i_{1}, i_{2}$, and $i_{3}$, respectively.)

Using (2.6), we find the abstract forms of all the terms involved in (3.18). More precisely, we have

$$
\begin{gathered}
u(i+1, j, n)=\left(u, e_{i+1, j, n}\right)=\left(u, V_{1} e_{i, j, n}\right)=\left(V_{1}^{*} u, e_{i, j, n}\right), \\
u(i, j+1, n)=\left(u, e_{i, j+1, n}\right)=\left(u, V_{2} e_{i, j, n}\right)=\left(V_{2}^{*} u, e_{i, j, n}\right), \\
u(i, j, n+1)=\left(u, e_{i, j, n+1}\right)=\left(u, V_{3} e_{i, j, n}\right)=\left(V_{3}^{*} u, e_{i, j, n}\right), \\
u(i-1, j, n)=\left(u, e_{i-1, j, n}\right)=\left(u, V_{1}^{*} e_{i, j, n}\right)=\left(V_{1} u, e_{i, j, n}\right), \\
u(i, j-1, n)=\left(u, e_{i, j-1, n}\right)=\left(u, V_{2}^{*} e_{i, j, n}\right)=\left(V_{2} u, e_{i, j, n}\right), \\
b(i, j, n) u(i, j, n)=\left(B u, e_{i, j, n}\right),
\end{gathered}
$$

where $B$ is the diagonal operator $B e_{i, j, n}=b(i, j, n) e_{i, j, n}$ for a sequence $b(i, j, n)$. Thus, the abstract form of (3.18) in $H$ is

$$
V_{3}^{*} u+R_{1} u-R V_{1} u-R V_{1}^{*} u-R V_{2} u-R V_{2}^{*} u=0
$$

where $R, R_{1}$ are the diagonal operators

$$
R e_{i, j, n}=r(i, j, n) e_{i, j, n}, \quad R_{1} e_{i, j, n}=[4 r(i, j, n)-1] e_{i, j, n}, \quad i, j, n \geq 1
$$

(a) Due to (3.20), (3.25) is rewritten as follows:

$$
(I-T) u=0,
$$

where $T=-R_{1}^{-1} V_{3}^{*}+R_{1}^{-1} R V_{1}+R_{1}^{-1} R V_{1}^{*}+R_{1}^{-1} R V_{2}+R_{1}^{-1} R V_{2}^{*}$. But $\|T\| \leq\left\|R_{1}^{-1}\right\|(1+$ $4\|R\|)<1$ due to (3.21). Thus, according to Theorem 1.1, the inverse of $I-T$ exists and is a linear bounded operator in $H$. Thus, the unique solution of (3.27) in $H$ is the zero solution. Equivalently, this means that the unique solution of (3.18) in $\ell_{3}^{2}$ is the zero solution.

(b) Since $V_{3}^{*} e_{i, j, 1}=0,(3.25)$ is written as follows:

$$
(I-T) u=\sum_{i=1}^{\infty} \sum_{j=1}^{\infty} u(i, j, 1) e_{i, j, 1}
$$


where $T=-V_{3} R_{1}+V_{3} R V_{1}+V_{3} R V_{1}^{*}+V_{3} R V_{2}+V_{3} R V_{2}^{*}$. But $\|T\| \leq\left\|R_{1}\right\|+4\|R\|<1$ due to (3.22). Thus, the inverse of $I-T$ exists and is a linear operator of $H$ bounded by

$$
\left\|(I-T)^{-1}\right\| \leq \frac{1}{1-\sup _{i, j, n}|4 r(i, j, n)-1|-4 \sup _{i, j, n}|r(i, j, n)|} .
$$

Thus, (3.28) has a unique solution in $H$ bounded by

$$
\|u\| \leq \frac{\left\|\sum_{i=1}^{\infty} \sum_{j=1}^{\infty} u(i, j, 1) e_{i, j, 1}\right\|}{1-\sup _{i, j, n}|4 r(i, j, n)-1|-4 \sup _{i, j, n}|r(i, j, n)|} .
$$

Equivalently, this means that (3.18) has a unique solution in $\ell_{3}^{2}$, which satisfies (3.23).

Remark 3.4. (a) Since $u(i, j, n) \in \ell_{3}^{2}$, we have $\lim _{i, j, n \rightarrow \infty} u(i, j, n)=0$. The physical importance of this fact is that after a long period of time (theoretically infinite), at the end of the plate (which is assumed to be of infinite length), the temperature will tend to zero, which is in agreement with the physical laws.

(b) In [2], (3.18) is mentioned but not studied. More precisely, it is stated there that if the plate has an initial temperature at $n=0$, then after a quite large time interval, the temperature of the plate will not depend on time, but only on the position $(i, j)$. When this happens, the temperature $u(i, j)$ of the plate will satisfy the linear, homogeneous partial difference equation of two variables, which is characterized as the steady state equation

$$
u(i-1, j)+u(i+1, j)+u(i, j-1)+u(i, j+1)-4 u(i, j)=0
$$

This equation has a positive, bounded solution which is $u(i, j) \equiv 1$. (Note that this solution does not belong to $\ell_{2}^{2}$.) Then an important question is the following [2].

"Do equations of the form

$$
\begin{gathered}
\alpha(i, j) u(i-1, j)+\beta(i, j) u(i+1, j)+\gamma(i, j) u(i, j-1) \\
+\delta(i, j) u(i, j+1)-\sigma(i, j) u(i, j)=0
\end{gathered}
$$

where $\alpha(i, j), \beta(i, j), \gamma(i, j), \delta(i, j)$, and $\sigma(i, j)$ are real sequences, have bounded and/or positive solutions?"

The following was proved in [2]: if $\alpha(i, j), \beta(i, j), \gamma(i, j), \delta(i, j)$, and $\sigma(i, j)$ are positive sequences with

$$
\sup _{i, j}\left\{\left|\frac{\alpha(i, j)}{\sigma(i, j)}\right|+\left|\frac{\beta(i, j)}{\sigma(i, j)}\right|+\left|\frac{\gamma(i, j)}{\sigma(i, j)}\right|+\left|\frac{\delta(i, j)}{\sigma(i, j)}\right|\right\}<1,
$$

then the unique bounded solution of (3.32) with $i, j=0, \pm 1, \pm 2, \ldots$ is the zero solution. 
In a way similar to the proof of Result 3.3, we can prove the following.

(i) If

$$
\sup _{i, j}\left|\frac{\alpha(i, j)}{\sigma(i, j)}\right|+\sup _{i, j}\left|\frac{\beta(i, j)}{\sigma(i, j)}\right|+\sup _{i, j}\left|\frac{\gamma(i, j)}{\sigma(i, j)}\right|+\sup _{i, j}\left|\frac{\delta(i, j)}{\sigma(i, j)}\right|<1
$$

then the unique bounded solution of (3.32) in $\ell_{2}^{2}$ is the zero solution.

Note that (3.34) implies (3.33).

(ii) If $u(i, 1) \in \ell_{1}^{2}$ and

$$
\sup _{i, j}\left|\frac{\alpha(i, j)}{\delta(i, j)}\right|+\sup _{i, j}\left|\frac{\beta(i, j)}{\delta(i, j)}\right|+\sup _{i, j}\left|\frac{\gamma(i, j)}{\delta(i, j)}\right|+\sup _{i, j}\left|\frac{\sigma(i, j)}{\delta(i, j)}\right|<1,
$$

then (3.32) has a unique bounded solution in $\ell_{2}^{2}$, which satisfies

$$
|u(i, j)| \leq \frac{\|u(i, 1)\|_{\mathbb{N}_{\mathbb{N}}^{2}}}{1-\sup _{i, j}\left|\frac{\alpha(i, j)}{\delta(i, j)}\right|-\sup _{i, j}\left|\frac{\beta(i, j)}{\delta(i, j)}\right|-\sup _{i, j}\left|\frac{\gamma(i, j)}{\delta(i, j)}\right|-\sup _{i, j}\left|\frac{\sigma(i, j)}{\delta(i, j)}\right|} .
$$

(iii) If $u(1, j) \in \ell_{1}^{2}$ and

$$
\sup _{i, j}\left|\frac{\alpha(i, j)}{\beta(i, j)}\right|+\sup _{i, j}\left|\frac{\gamma(i, j)}{\beta(i, j)}\right|+\sup _{i, j}\left|\frac{\delta(i, j)}{\beta(i, j)}\right|+\sup _{i, j}\left|\frac{\sigma(i, j)}{\beta(i, j)}\right|<1,
$$

then (3.32) has a unique bounded solution in $\ell_{2}^{2}$, which satisfies

$$
|u(i, j)| \leq \frac{|| u(1, j) \|_{\ell_{\mathbb{N}}^{2}}}{1-\sup _{i, j}\left|\frac{\alpha(i, j)}{\beta(i, j)}\right|-\sup _{i, j}\left|\frac{\gamma(i, j)}{\beta(i, j)}\right|-\sup _{i, j}\left|\frac{\delta(i, j)}{\beta(i, j)}\right|-\sup _{i, j}\left|\frac{\sigma(i, j)}{\beta(i, j)}\right|} .
$$

\section{Acknowledgment}

The authors would like to thank the referees for their remarks which helped to improve the presentation of this paper.

\section{References}

[1] L. E. Bush, The William Lowell Putnam mathematical competition, Amer. Math. Monthly 72 (1965), no. 7, 732-739.

[2] S. S. Cheng and R. Medina, Bounded and positive solutions of discrete steady state equations, Tamkang J. Math. 31 (2000), no. 2, 131-135.

[3] C. J. Earle and R. S. Hamilton, A fixed point theorem for holomorphic mappings, Global Analysis (Proc. Sympos. Pure Math., Vol. XVI, Berkeley, Calif, 1968), American Mathematical Society, Rhode Island, 1970, pp. 61-65.

[4] I. Gohberg and S. Goldberg, Basic Operator Theory, Birkhäuser Boston, Massachusetts, 1981.

[5] E. K. Ifantis, On the convergence of power series whose coefficients satisfy a Poincaré-type linear and nonlinear difference equation, Complex Variables Theory Appl. 9 (1987), no. 1, 63-80. 


\section{Functional-analytic method for difference equations}

[6] N. Kruse and T. Nesemann, Global asymptotic stability in some discrete dynamical systems, J. Math. Anal. Appl. 235 (1999), no. 1, 151-158.

[7] E. N. Petropoulou, On some specific non-linear ordinary difference equations, Arch. Math. (Brno) 36 (2000), 549-562.

[8] , Partial difference equations arising in numerical schemes and game theory, Appl. Math. Comput. 141 (2003), no. 1, 185-196.

[9] E. N. Petropoulou and P. D. Siafarikas, Bounded solutions and asymptotic stability of nonlinear difference equations in the complex plane, Arch. Math. (Brno) 36 (2000), no. 2, 139-158.

[10] Bounded solutions and asymptotic stability of nonlinear difference equations in the complex plane. II, Comput. Math. Appl. 42 (2001), no. 3-5, 427-452.

[11] Bounded solutions of a class of linear delay and advanced partial difference equations, Dynam. Systems Appl. 10 (2001), no. 2, 243-260.

[12] Solutions of nonlinear delay and advanced partial difference equations in the space $l_{\mathbb{N} \times \mathbb{N}}^{1}$, Comput. Math. Appl. 45 (2003), no. 6-9, 905-934.

Eugenia N. Petropoulou: Department of Engineering Sciences, Division of Applied Mathematics and Mechanics, University of Patras, 26500 Patras, Greece

E-mail address: jenpetro@des.upatras.gr

Panayiotis D. Siafarikas: Department of Mathematics, University of Patras, 26500 Patras, Greece E-mail address: panos@math.upatras.gr 